Proceedings of the Edinburgh Mathematical Society (2006) 49, 739-751 (C)

DOI:10.1017/S0013091505000040 Printed in the United Kingdom

\title{
ON CONNECTIONS BETWEEN DELTA-CONVEX MAPPINGS AND CONVEX OPERATORS
}

\author{
LIBOR VESELÝ ${ }^{1}$ AND LUDĚK ZAJÍČEK ${ }^{2}$ \\ ${ }^{1}$ Dipartimento di Matematica, Università degli Studi, Via C. Saldini 50, \\ 20133 Milano, Italy (vesely@mat.unimi.it) \\ ${ }^{2}$ Charles University, Faculty of Mathematics and Physics, Sokolovská 83, \\ 18675 Praha 8, Czech Republic (zajicek@karlin.mff.cuni.cz)
}

(Received 21 January 2005)

\begin{abstract}
We study conditions under which every delta-convex (d.c.) mapping is the difference of two continuous convex operators, and vice versa. In particular, we prove that each d.c. mapping $F:(a, b) \rightarrow Y$ is the difference of two continuous convex operators whenever $Y$ belongs to a large class of Banach lattices which includes all $L^{p}(\mu)$ spaces $(1 \leqslant p \leqslant \infty)$. The proof is based on a result about Jordan decomposition of vector-valued functions. New observations on Jordan decomposition of finitely additive vector-valued measures are also presented.
\end{abstract}

Keywords: delta-convex (d.c.) mapping; convex operator; Lipschitz condition; normed linear space; Banach lattice; Jordan decomposition

2000 Mathematics subject classification: Primary 47H99

Secondary 28B15

\section{Introduction}

Let $C$ be an open convex set in a (real) normed linear space $X$. A real-valued function $f$ on $C$ is delta-convex (d.c.) if it can be represented as the difference of two continuous convex functions on $C$.

We consider the following three natural generalizations of the notion of a d.c. function to mappings between normed spaces (the definitions of an ordered normed space and of a convex operator are recalled at the end of this section).

Definition 1.1. Let $X, Y$ be normed linear spaces, let $C \subset X$ be an open convex set, and let $F: C \rightarrow Y$ be a mapping.

(a) $F$ is a d.c. mapping (i.e. a delta-convex mapping) on $C$ if there exists a continuous convex function $f: C \rightarrow \mathbb{R}$ (control function) such that, for each $y^{*} \in Y^{*}$ with $\left\|y^{*}\right\| \leqslant 1$, the function $y^{*} \circ F+f$ is continuous and convex on $C$.

(b) $F$ is weakly d.c. on $C$ if $y^{*} \circ F$ is a d.c. function on $C$ for each $y^{*} \in Y^{*}$. 
(c) If $Y$ is an ordered normed space, we say that $F$ is order d.c. on $C$ if $F$ can be represented as the difference of two continuous convex operators on $C$.

Note that the notion of a d.c. mapping has many nice properties (see [11] and also [4]) and seems to be the 'most natural' generalization of d.c. functions. It is easy to see that the three notions from Definition 1.1 are equivalent for $Y=\mathbb{R}^{n}$ (equipped with the standard coordinate-wise partial ordering). In the present article we study relationships between these three classes of mapping in the case of an infinite-dimensional $Y$; in particular we improve some results of $[\mathbf{1 1}],[\mathbf{4}]$ and $[\mathbf{1 2}]$.

It follows immediately from definitions that each d.c. mapping is weakly d.c. Moreover (see Observation 2.1), if $Y$ is an ordered normed space whose positive cone is normal (which is the most interesting case, satisfied by all Banach lattices), then each order-d.c. mapping is also weakly d.c. Thus the class of weakly d.c. mappings is the widest one.

In $\S 2$ we observe that the Banach-Steinhaus theorem easily implies that $F: X \rightarrow Y$ is locally Lipschitz whenever $F$ is weakly locally Lipschitz (i.e. $y^{*} \circ F$ is locally Lipschitz for each $\left.y^{*} \in Y^{*}\right)$. This fact provides a simple unified proof of results on local Lipschitz continuity from [3] (for continuous convex operators) and [11] (for d.c. mappings).

In $\S 3$ we observe that the assertion 'd.c. implies order d.c.' is true for mappings into ordered normed spaces whose unit ball admits an upper bound (which holds for $L^{\infty}(\mu)$ and for $C(K)$ spaces). It was proved in [12] that the same assertion holds for mappings $F:(a, b) \rightarrow Y$, where $Y$ belongs to a large class of sequence spaces containing all $\ell^{p}(\Gamma)$ spaces. Here we generalize this result to the case when $Y$ is a Banach lattice with the $\sigma$-Levi property. Our proof is based on Theorem 3.7 about Jordan decomposition of a vector function $f:(a, b) \rightarrow Y$ of locally finite variation, which is interesting in its own right.

Let us remark that the implication 'd.c. implies order d.c.' does not hold [12] in general for mappings $F:(a, b) \rightarrow c_{0}$ and $F: \mathbb{R}^{d} \rightarrow \ell^{p}$, where $d \geqslant 2$ and $1 \leqslant p<d$.

The implication 'order d.c. implies d.c.' is not usually true. In $\S 4$ we show that this implication does not hold in general whenever $X$ is an arbitrary (non-trivial) Banach space and $Y$ is an infinite-dimensional $L^{p}(\mu)$ space $(1<p<\infty)$. This result (Proposition 4.3) generalizes Proposition 21 from [4], where $X$ is separable and $L^{p}(\mu)=l^{2}$. However, if the range space of an order-d.c. mapping is an ordered normed space whose positive cone is well-based (and this is true for $L^{1}(\mu)$ ), it is easy to show (see Proposition 4.1) that the mapping is then d.c.

Note that the above results imply that a mapping of one real variable with values in $L^{1}(\mu)$ is d.c. if and only if it is order d.c.

A special case of our Theorem 3.7, when $Y$ is a Nakano space, could be easily deduced from known results on Jordan decomposition of finitely additive vector-valued measures. Such a procedure could also be used in the case when $Y$ is only a Levi space. Indeed, we observe in $\S 5$ that the needed Jordan decomposition theorem for measures also holds for such spaces. However, there are natural examples of $\sigma$-Levi spaces which are not Levi (see Remark 3.8 (iii)).

Let us recall some terminology (quite unstandardized in the literature) concerning ordered normed spaces. 
By an ordered normed space we mean a normed linear space $Y$ that is equipped with an (antisymmetric) partial ordering ' $\leqslant$ ' such that, for $x, y, z \in Y$ and $\lambda \geqslant 0$, the implications $x \leqslant y \Rightarrow x+z \leqslant y+z$ and $x \leqslant y \Rightarrow \lambda x \leqslant \lambda y$ hold. Then the cone $Y_{+}:=\{y \in Y: y \geqslant 0\}$ is called the positive cone of $Y$. The dual $Y^{*}$ of an ordered normed space $Y$ is also an ordered normed space when equipped with the following partial ordering: $x^{*} \leqslant y^{*}$ if and only if $x^{*}(u) \leqslant y^{*}(u)$ for each $u \in Y_{+}$.

Let $C$ be an open convex subset of a normed linear space $X$ and let $Y$ be an ordered normed linear space. Then a mapping $G: C \rightarrow Y$ is called a convex operator if

$$
G((1-t) x+t y) \leqslant(1-t) G(x)+t G(y)
$$

whenever $x, y \in C$ and $0 \leqslant t \leqslant 1$.

We say that the positive cone $Y_{+}$is normal ('self-allied' in [6]) if there exists $\delta>0$ such that $\delta\|y\| \leqslant\|z\|$ whenever $0 \leqslant y \leqslant z$. (This is one of a number of equivalent definitions: see $\left[\mathbf{6}\right.$, p. 90].) If $Y_{+}$is normal, then $Y^{*}=Y_{+}^{*}-Y_{+}^{*}$ (see [6, Theorem 3.6.2]). (Note that the opposite implication, which we do not need, also holds.)

We say that a convex subset $B$ of an ordered normed space $Y$ is a base for the positive cone $Y_{+}$if, for each $y \in Y_{+} \backslash\{0\}$, there exists a unique $\lambda>0$ such that $\lambda y \in B$. Following [6, p. 120], we say that $Y_{+}$is well-based if it has a bounded base $B$ such that $0 \notin \bar{B}$. By [6], 3.8.12, $Y_{+}$is well-based if and only if there exists $\varphi \in Y^{*}$ such that $\varphi(u) \geqslant\|u\|$ for each $u \in Y_{+}$.

It is easy to see that

$$
B_{Y} \text { has an upper bound if and only if } Y_{+} \text {has an interior point. }
$$

Moreover, there is a simple duality between well-basedness and existence of interior points for $Y_{+}$and $Y_{+}^{*}($ see $[6,3.8 .12])$ :

$Y_{+}$is well-based if and only if $Y_{+}^{*}$ has an interior point;

if $Y_{+}$has an interior point, then $Y_{+}^{*}$ is well-based.

An ordered normed space $Y$ is a Banach lattice if it is a Banach space, each pair of elements of $Y$ has a supremum and an infimum, and $0 \leqslant|x| \leqslant|y|$ implies $\|x\| \leqslant\|y\|$ (where $|x|:=\sup (x,-x)$ ).

We use the following (quite usual) notation.

The closed unit ball of a normed linear space $Y$ is denoted by $B_{Y}$. The symbol $C_{b}(T)$ (respectively, $C(K)$ ) denotes the space of all continuous bounded functions on an arbitrary topological space $T$ (respectively, of all continuous functions on an arbitrary compact space $K$ ) equipped with the supremum norm. When we deal with the spaces $L^{p}(\mu)$, we allow an arbitrary measure $\mu$.

All normed linear spaces are real.

\section{The Lipschitz property of weakly d.c. mappings and its consequences}

Obviously, each d.c. mapping is weakly d.c. For order-d.c. mappings we have the following observation. 
Observation 2.1. Let $Y$ be an ordered normed space whose positive cone is normal. Then each continuous convex operator $F$ into $Y$ (and, consequently, also each order-d.c. mapping into $Y$ ) is weakly d.c. (Indeed, since $Y_{+}$is normal, each $y^{*} \in Y^{*}$ can be written as $y^{*}=u^{*}-v^{*}$ with $u^{*}, v^{*} \in Y_{+}$; hence $y^{*} \circ F=u^{*} \circ F-v^{*} \circ F$ is a d.c. function.)

It is possible that the following simple result is known, but we have been unable to find it in the literature.

Proposition 2.2. Let $X, Y$ be normed linear spaces, let $C \subset X$ be an open set, and let $F: C \rightarrow Y$ be weakly locally Lipschitz (i.e. $y^{*} \circ F$ is locally Lipschitz for each $\left.y^{*} \in Y^{*}\right)$. Then $F$ is locally Lipschitz on $C$.

Proof. Let $a \in C$ be given. For each natural number $n$ denote by $Z_{n}$ the set of all functionals $y^{*} \in Y^{*}$ for which the function $y^{*} \circ F$ is Lipschitz on the set $C \cap B(a, 1 / n)$. Obviously, $\bigcup_{n=1}^{\infty} Z_{n}=Y^{*}$ and we can choose $n$ such that $Z_{n}$ is a set of the second category in $Y^{*}$. Since the set $M \subset Y \subset Y^{* *}$,

$$
M:=\left\{\frac{F(x)-F(y)}{\|x-y\|}: x, y \in B(a, 1 / n) \cap C, x \neq y\right\},
$$

is pointwise bounded on $Z_{n}$, the Banach-Steinhaus theorem implies that $M$ is norm bounded in $Y^{* *}$. In other words, $F$ is Lipschitz on $B(a, 1 / n) \cap C$.

\section{Corollary 2.3.}

(a) Each weakly d.c. mapping is locally Lipschitz. In particular, each d.c. mapping is locally Lipschitz (which was first proved in [11]).

(b) If $Y$ is an ordered normed space whose positive cone is normal, then each continuous convex operator (and hence also each order-d.c. mapping) into $Y$ is locally Lipschitz (which was first proved in $[\mathbf{3}]$ ).

\section{When d.c. implies order d.c.}

We start with the following simple observation.

Proposition 3.1. Let $Y$ be an ordered normed space whose closed unit ball has an upper bound. Then each d.c. mapping into $Y$ is order d.c.

Proof. Let $e \in Y$ be an upper bound for $B_{Y}$, i.e. $y \leqslant\|y\| e$ for each $y \in Y$. Let $C \subset X$ be an open convex set, and let $F: C \rightarrow Y$ be a d.c. mapping with a control function $f$. Then the mapping $G(x)=f(x) e$ is a continuous convex operator and, for each $x, y \in C$ and $0 \leqslant t \leqslant 1$,

$$
\begin{aligned}
(1-t) F(x)+t F(y)-F((1-t) x+t y) & \leqslant\|(1-t) F(x)+t F(y)-F((1-t) x+t y)\| e \\
& \leqslant(1-t) G(x)+t G(y)-G((1-t) x+t y) .
\end{aligned}
$$


Here we have used the inequality (see [11, Proposition 1.13])

$$
\|(1-t) F(x)+t F(y)-F((1-t) x+t y)\| \leqslant(1-t) f(x)+t f(y)-f((1-t) x+t y) .
$$

Hence $H:=G-F$ is a continuous convex operator. Thus $F=G-H$ is order d.c.

Corollary 3.2. Each d.c. mapping into any of the spaces $C_{b}(T), L^{\infty}(\mu), C_{b}(T)^{* *}$ or $L^{\infty}(\mu)^{* *}$ is order d.c.

Proof. It is obvious that the constant function $f=1$ is an upper bound for the unit ball both in $C_{b}(T)$ and in $L^{\infty}(\mu)$. Using (1.1), (1.2) and (1.3), we obtain the rest of the statement.

Now we are going to show that, for a large class of Banach lattices $Y$ (those which have the $\sigma$-Levi property), every d.c. mapping of one real variable with values in $Y$ is order d.c. (Theorem 3.11). We do this via proving a result about Jordan decomposition of mappings with locally finite variation.

Let us start with a definition of the $\sigma$-Levi property. Recall that a partially ordered set $M$ is called upper directed if each finite subset of $M$ has an upper bound in $M$.

Definition 3.3. Let $X$ be a Banach lattice. We shall say that $X$ has

(a) the Levi property if each norm-bounded upper-directed set in $X_{+}$has a least upper bound (cf. [2]; the term 'monotonically complete' was used in [8]);

(b) the $\sigma$-Levi property if each norm-bounded non-decreasing sequence in $X_{+}$has a least upper bound (cf. [2]).

The following observation explains the terminology ' $\sigma$-Levi property'.

Observation 3.4. A Banach lattice $X$ has the $\sigma$-Levi property if and only if each countable norm-bounded upper-directed set in $X_{+}$has a least upper bound. (One implication is obvious. On the other hand, if $X$ is $\sigma$-Levi and $M=\left\{x_{n}\right\} \subset X_{+}$is norm bounded and upper directed, it is easy to see that the points $y_{n}:=\sup \left\{x_{1}, \ldots, x_{n}\right\}$ form a norm-bounded non-decreasing sequence in $X_{+}$. Moreover, the least upper bound of $\left\{y_{n}\right\}$ is the least upper bound for $M$ as well.)

The following lemma is an easy variant of Proposition 2.4.19(i) in [8].

Lemma 3.5. Let $X$ be a Banach lattice having the $\sigma$-Levi property. Then there exists $r>0$ such that, for each norm-bounded non-decreasing sequence $\left\{x_{i}\right\} \subset X_{+}$, one has $\left\|\sup _{i} x_{i}\right\| \leqslant r \sup _{i}\left\|x_{i}\right\|$.

Proof. If not, for each positive integer $n$ there exists a norm-bounded non-decreasing sequence $\left\{x_{i}^{(n)}\right\}_{i=1}^{\infty} \subset X_{+}$such that

$$
\left\|\sup _{i} x_{i}^{(n)}\right\|>n^{3} \sup _{i}\left\|x_{i}^{(n)}\right\| \quad \text { and } \quad\left\|\sup _{i} x_{i}^{(n)}\right\|=n .
$$


Hence we have $\left\|x_{i}^{(n)}\right\| \leqslant\left(1 / n^{2}\right)$ for all $i$ and $n$. Put

$$
M:=\left\{\sup F: F \subset\left\{x_{i}^{(n)}\right\}_{i, n=1}^{\infty} \text { finite }\right\} .
$$

Then $M$ is a countable and upper-directed subset of $X_{+}$.

We claim that $M$ is norm bounded. Let $z \in M$ be given. Since each $\left\{x_{i}^{(n)}\right\}_{i=1}^{\infty}$ is non-decreasing, we can write

$$
z=\sup \left\{x_{i_{1}}^{\left(n_{1}\right)}, \ldots, x_{i_{k}}^{\left(n_{k}\right)}\right\}
$$

where the integers $n_{j}$ are pairwise distinct. Then $0 \leqslant z \leqslant \sum_{j=1}^{k} x_{i_{j}}^{\left(n_{j}\right)}$ implies

$$
\|z\| \leqslant \sum_{j=1}^{k}\left\|x_{i_{j}}^{\left(n_{j}\right)}\right\| \leqslant \sum_{1}^{\infty} \frac{1}{n^{2}} .
$$

The claim is proved.

By Observation 3.4, there exists $\sup M \in X$. Then, for each $n$, we have $\sup M \geqslant$ $\sup _{i} x_{i}^{(n)} \geqslant 0$, which implies that $\|\sup M\| \geqslant\left\|\sup _{i} x_{i}^{(n)}\right\|=n$. But this is impossible.

We shall need the following properties of mappings of finite variation.

Fact 3.6. Let $[a, b] \subset \mathbb{R}$ be a non-degenerate interval, let $Y$ be a Banach space, and let $f:[a, b] \rightarrow Y$ be a mapping with finite variation, i.e.

$$
\operatorname{var}(a, b):=\sup _{P}\left\{\sum_{i=1}^{n}\left\|f\left(t_{i}\right)-f\left(t_{i-1}\right)\right\|\right\}<\infty,
$$

where the supremum is taken over all partitions $P=\left\{a=t_{0}<t_{1}<\cdots<t_{n}=b\right\}$ of $[a, b]$.

Then

(a) $f$ has at most countably many points of discontinuity in $[a, b]$;

(b) $\operatorname{var}(a, b)=\operatorname{var}(a, c)+\operatorname{var}(c, b)$ whenever $a \leqslant c \leqslant b$;

(c) $f$ is Bochner integrable on $[a, b]$.

Proof. By [5, 2.5.16], $f$ satisfies (a), (b) and has a separable range. Moreover, (a) implies that $f$ is Borel measurable. Since $f$ is also bounded, we conclude that (c) holds (see, for example, $[\mathbf{1}, 11.36,11.38]$ ).

Theorem 3.7. Let $I \subset \mathbb{R}$ be an open interval, let $Y$ be a Banach lattice with the $\sigma$-Levi property, and let $f: I \rightarrow Y$ be a mapping having locally finite variation.

Then there exist non-decreasing mappings $g, h: I \rightarrow Y$ such that $f=g-h$ and $g, h$ have locally finite variation. Moreover, the decomposition $f=g-h$ is minimal in the class of all representations of $f$ as the difference of non-decreasing mappings, i.e. if $f=g^{*}-h^{*}$ is such a representation, then $g(\beta)-g(\alpha) \leqslant g^{*}(\beta)-g^{*}(\alpha)$ for all $\alpha<\beta$, $\alpha, \beta \in I$. 
Proof. As usual, we use the notation $y^{+}:=\sup \{y, 0\}(y \in Y)$. First, we are going to show that, for each non-degenerate interval $[a, b] \subset I$, there exists the 'positive-order variation'

$$
V^{+}(a, b):=\sup _{P}\{S(P)\} \in Y,
$$

where the least upper bound (in $Y$ ) is taken over all partitions $P=\left\{a=t_{0}<t_{1}<\cdots<\right.$ $\left.t_{k}=b\right\}$ of $[a, b]$, and

$$
S(P)=\sum_{i=1}^{k}\left[f\left(t_{i}\right)-f\left(t_{i-1}\right)\right]^{+} .
$$

Then, as in the classical scalar case, we shall show that one minimal decomposition $f=g-h$ can be constructed by fixing a point $c \in I$ and putting

$$
g(t)= \begin{cases}V^{+}(c, t) & \text { if } t>c \\ 0 & \text { if } t=c \\ -V^{+}(t, c) & \text { if } t<c\end{cases}
$$

By Fact 3.6, the set $D$ of all points of discontinuity of $f$ is at most countable. Fix a non-degenerate interval $[a, b] \subset I$. Let $\left\{P_{n}\right\}$ be an increasing sequence of partitions of $[a, b]$ such that the set $\tilde{D}:=\bigcup_{n=1}^{\infty} P_{n}$ is dense in $[a, b]$ and contains $D \cap[a, b]$. Clearly, $S\left(P_{1}\right) \leqslant S\left(P_{2}\right) \leqslant S\left(P_{3}\right) \leqslant \cdots$. Moreover, it is easy to see that $\left\|S\left(P_{n}\right)\right\| \leqslant \operatorname{var}(a, b)$ for all $n$, where $\operatorname{var}(a, b)$ denotes the usual (norm) variation of $f$ on $[a, b]$ as in Fact 3.6. By the $\sigma$-Levi property, there exists $s:=\sup _{n} S\left(P_{n}\right) \in Y$ and

$$
\|s\| \leqslant r \cdot \operatorname{var}(a, b)
$$

where $r$ is as in Lemma 3.5. To prove that $s=V^{+}(a, b)$, it is clearly sufficient to show that $S(P) \leqslant s$ for each partition $P=\left\{a=t_{0}<t_{1}<\cdots<t_{k}=b\right\}$ of $[a, b]$.

Let $E$ be the set of the indices $i \in\{0, \ldots, k\}$ such that $t_{i} \in P \backslash \tilde{D}$. Fix $\varepsilon>0$. It is easy to see that, taking $n$ sufficiently large, the partition $P_{n}=\left\{a=x_{0}<x_{1}<\cdots<x_{m}=b\right\}$ has the following properties:

(a) $P \cap \tilde{D} \subset P_{n}$;

(b) there exists an increasing function $j: E \rightarrow\{0, \ldots, m-1\}$ such that, for each $i \in E$, one has

$$
x_{j(i)}<t_{i}<x_{j(i)+1} \quad \text { and } \quad\left\|f\left(t_{i}\right)-f\left(x_{j(i)}\right)\right\|+\left\|f\left(x_{j(i)+1}\right)-f\left(t_{i}\right)\right\| \leqslant \frac{\varepsilon}{k}
$$

(recall that $f$ is continuous at each $t_{i}, i \in E$ ).

Then

$$
S(P) \leqslant S\left(P \cup P_{n}\right) \leqslant S\left(P_{n}\right)+\sum_{i \in E}\left(\left[f\left(t_{i}\right)-f\left(x_{j(i)}\right)\right]^{+}+\left[f\left(x_{j(i)+1}\right)-f\left(t_{i}\right)\right]^{+}\right) .
$$


Consequently, $S(P) \leqslant s+z_{\varepsilon}$ where $z_{\varepsilon}$ is the sum from the preceding formula. Since

$$
\left\|z_{\varepsilon}\right\| \leqslant \sum_{i \in E}\left(\left\|f\left(t_{i}\right)-f\left(x_{j(i)}\right)\right\|+\left\|f\left(x_{j(i)+1}\right)-f\left(t_{i}\right)\right\|\right) \leqslant \varepsilon
$$

and the positive cone of each Banach lattice is closed (cf. Proposition 1.1.6 in [8]), we can conclude that $S(P) \leqslant s$.

We have proved that the positive-order variation $V^{+}(a, b)$ of $f$ exists for each nondegenerate interval $[a, b] \subset I$. Exactly as in the scalar case, one can prove that $[a, b] \mapsto$ $V^{+}(a, b)$ is a non-negative additive interval function with values in $Y$. Thus, clearly, the interval function $[a, b] \mapsto V^{+}(a, b)-[f(b)-f(a)]$ is also non-negative and additive. Consequently, the mappings $g$ (from (3.1)) and $h:=g-f$ are non-decreasing. Moreover, $g$ (and consequently also $h$ ) has bounded variation on each $[a, b] \subset I$ since, for every partition $P=\left\{a=t_{0}<\cdots<t_{k}=b\right\}$, we have by $(3.2)$

$$
\sum_{i=1}^{n}\left\|g\left(t_{i}\right)-g\left(t_{i-1}\right)\right\|=\sum_{i=1}^{n}\left\|V^{+}\left(t_{i-1}, t_{i}\right)\right\| \leqslant r \sum_{i=1}^{n} \operatorname{var}\left(t_{i-1}, t_{i}\right)=r \cdot \operatorname{var}(a, b) .
$$

It remains to prove that our decomposition $f=g-h$ is minimal. Let $f=g^{*}-h^{*}$ be another representation of $f$ as the difference of non-decreasing mappings with locally finite variation. Fix $\alpha, \beta \in I$ such that $\alpha<\beta$. Then for every partition $P=\left\{\alpha=t_{0}<\right.$ $\left.\cdots<t_{n}=\beta\right\}$ of $[\alpha, \beta]$ we have

$$
\begin{aligned}
S(P) & =\sum_{i=1}^{n}\left[g^{*}\left(t_{i}\right)-g^{*}\left(t_{i-1}\right)-h^{*}\left(t_{i}\right)+h^{*}\left(t_{i-1}\right)\right]^{+} \\
& \leqslant \sum_{i=1}^{n}\left[g^{*}\left(t_{i}\right)-g^{*}\left(t_{i-1}\right)\right]^{+} \\
& =g^{*}(\beta)-g^{*}(\alpha),
\end{aligned}
$$

which implies that $g(\beta)-g(\alpha)=V^{+}(\alpha, \beta) \leqslant g^{*}(\beta)-g^{*}(\alpha)$.

\section{Remark 3.8.}

(i) Recall that a Nakano space is a Banach lattice in which every norm-bounded upperdirected set $M$ has a supremum satisfying $\|\sup M\|=\sup _{x \in M}\|x\|$. The particular case of Theorem 3.7 when $Y$ is a Nakano space could also be proved using known results $[\mathbf{7}, \mathbf{1 0}]$ on Jordan decomposition of finitely additive vector measures. Such a procedure could also be used in a more general case of a Levi space $Y$, since we could use Theorem 5.1 below. Of course, a direct proof is also quite easy in these cases, since the existence of the positive-order variation $V^{+}(a, b)=\sup \{S(P)\}$ follows immediately $(\{S(P)\}$ is an upper-directed set!) and the rest of the proof is rather standard.

(ii) The following Banach lattices are Nakano spaces (and hence satisfy the $\sigma$-Levi property). 
(a) All dual Banach lattices (cf. Proposition 2.4.19(ii) in [8]); in particular $L_{p}(\mu)$ $\left(1<p<\infty, \mu\right.$ arbitrary), $\ell_{\infty}(\Gamma)$ for any set $\Gamma, L_{\infty}(\mu)$ when $\mu$ is $\sigma$-finite.

(b) The Banach lattices $Y$ having the property (P) (i.e. there exists a positive linear projection of norm one from $Y^{* *}$ onto $Y$; this follows easily from (a)); in particular all AL-spaces (cf. [9]), e.g. $L_{1}(\mu)$ for any $\mu$.

(iii) There exist Banach lattices that have the $\sigma$-Levi property that are not Levi spaces (and so they are not Nakano spaces). For instance, consider the space of all bounded Borel functions on $\mathbb{R}$ with the supremum norm.

(iv) A version of Theorem 3.7, in which $I$ is a closed interval and $f, g, h$ have finite variation, also holds. The proof needs only obvious changes.

Before stating our theorem, we shall need the following two simple lemmas.

Lemma 3.9. Let $I \subset \mathbb{R}$ be an open interval, let $c$ be a point of $I$, and let $Y$ be a Banach lattice. Let $g: I \rightarrow Y$ be a non-decreasing mapping that is locally Bochner integrable on $I$. Then the mapping $G: I \rightarrow Y$, given by

$$
G(t)=\int_{c}^{t} g(\tau) \mathrm{d} t
$$

is a continuous convex operator.

Proof. It is well known that the indefinite Bochner integral is continuous. Since, for each $y^{*} \in Y_{+}^{*}$, the mapping $\tau \mapsto\left\langle g(\tau), y^{*}\right\rangle$ is non-decreasing, the function

$$
t \mapsto\left\langle G(t), y^{*}\right\rangle=\int_{c}^{t}\left\langle g(\tau), y^{*}\right\rangle \mathrm{d} \tau
$$

is clearly convex. Consequently, for each $t_{1}, t_{2} \in I$ and $\lambda \in[0,1]$,

$$
\left\langle(1-\lambda) G\left(t_{1}\right)+\lambda G\left(t_{2}\right)-G\left((1-\lambda) t_{1}+\lambda t_{2}\right), y^{*}\right\rangle \geqslant 0
$$

whenever $y^{*} \in Y_{+}^{*}$. It follows that $(1-\lambda) G\left(t_{1}\right)+\lambda G\left(t_{2}\right)-G\left((1-\lambda) t_{1}+\lambda t_{2}\right) \geqslant 0$ (cf. $[8,1.4 .2])$.

Lemma 3.10. Let $I \subset \mathbb{R}$ be an open interval, let $Y$ be a Banach space, and let $F: I \rightarrow Y$ be a d.c. mapping on $I$. Then the mapping $t \mapsto F_{+}^{\prime}(t)$ is everywhere defined and locally Bochner integrable in $I$. Moreover, for each $a, b \in I$,

$$
F(b)-F(a)=\int_{a}^{b} F_{+}^{\prime}(t) \mathrm{d} t .
$$

Proof. By Theorem 2.3 in $[\mathbf{1 1}], t \mapsto F_{+}^{\prime}(t)$ is everywhere defined and of locally bounded variation on $I$. By Fact 3.6, the Bochner integral $\int_{a}^{b} F_{+}^{\prime}(t) \mathrm{d} t$ exists for each 
$a, b \in I$. Moreover, $F$ is locally Lipschitz on $I$ by Corollary 2.3 ; hence, for each $y^{*} \in Y^{*}$, we have

$$
\begin{aligned}
\left\langle F(b)-F(a), y^{*}\right\rangle & =y^{*} \circ F(b)-y^{*} \circ F(a)=\int_{a}^{b}\left(y^{*} \circ F\right)^{\prime}(t) \mathrm{d} t \\
& =\int_{a}^{b}\left\langle F_{+}^{\prime}(t), y^{*}\right\rangle \mathrm{d} t=\left\langle\int_{a}^{b} F_{+}^{\prime}(t) \mathrm{d} t, y^{*}\right\rangle,
\end{aligned}
$$

which proves our formula.

Theorem 3.11. Let $I \subset \mathbb{R}$ be an open interval and let $Y$ be a Banach lattice having the $\sigma$-Levi property. Then each d.c. mapping $F: I \rightarrow Y$ is order d.c.

Proof. By Theorem 2.3 in [11], the right derivative $F_{+}^{\prime}(t)$ exists at each $t \in I$, and the mapping $F_{+}^{\prime}$ has locally bounded variation. By Theorem 3.7, we can write $F_{+}^{\prime}=g-h$ where $g, h$ are non-decreasing mappings with locally bounded variation on $I$. By Fact 3.6, $g$ and $h$ are locally Bochner integrable. Fix $c \in I$ and put

$$
G(t)=\int_{c}^{t} g(\tau) \mathrm{d} \tau, \quad H(t)=\int_{c}^{t} h(\tau) \mathrm{d} \tau .
$$

By Lemma 3.9, $G$ and $H$ are continuous convex operators. Using Lemma 3.10, we get

$$
F(t)=F(c)+\int_{c}^{t} F_{+}^{\prime}(t) \mathrm{d} t=(F(c)+G(t))-H(t),
$$

which completes the proof.

\section{When order d.c. implies d.c.}

The following easy proposition is the only positive result of this sort that we know. Note the duality between the assumptions in Proposition 3.1 and Proposition 4.1 (see (1.1), (1.2) and (1.3) in $\S 1)$.

Proposition 4.1. Let $Y$ be an ordered normed space whose positive cone is wellbased. Then each order-d.c. mapping into $Y$ is d.c.

Proof. Let $C$ be an open convex subset of a normed linear space $X$ and let $F: C \rightarrow Y$ be a continuous convex operator. Since $Y_{+}$is well-based, there exists $\varphi \in Y^{*}$ such that $\varphi(u) \geqslant\|u\|$ for each $u \in Y_{+}($see $\S 1)$. Then, for each $x, y \in C$,

$$
\left\|\frac{1}{2} F(x)+\frac{1}{2} F(y)-F\left(\frac{1}{2}(x+y)\right)\right\| \leqslant \varphi\left(\frac{1}{2} F(x)+\frac{1}{2} F(y)-F\left(\frac{1}{2}(x+y)\right)\right),
$$

which implies that $F$ is controlled by the function $\varphi \circ F$ (see [11, Corollary 1.18]). Since the difference of two d.c. mappings is d.c., the proof is complete.

Corollary 4.2. Let $Y$ be any of the spaces $L^{1}(\mu), L^{\infty}(\mu)^{*}, C_{b}(T)^{*}$. Then each orderd.c. mapping into $Y$ is d.c. 
Proof. The set $B:=\left\{f \in L^{1}(\mu): f \geqslant 0, \int f \mathrm{~d} \mu=1\right\}$ is clearly a bounded base for $\left(L^{1}(\mu)\right)_{+}$and $0 \notin \bar{B}$. The rest clearly follows from (1.1) and (1.3) (cf. the proof of Corollary 3.2).

The following proposition extends Proposition 21 in [4], where only mappings $F: X \rightarrow$ $\ell_{2}$ with $X$ separable were considered. We shall briefly say that a mapping is nowhere d.c. if it is d.c. on no open convex set.

Proposition 4.3. For every (non-trivial) Banach space $X$ and every infinite-dimensional $L_{p}(\mu)$ space with $1<p<\infty$, there exists an order-d.c. mapping $F: X \rightarrow L_{p}(\mu)$ which is nowhere d.c.

Proof. In the proof we will use the easy fact that the composition $\left(A_{1} \circ F\right.$ or $\left.F \circ A_{2}\right)$ of a d.c. mapping $F$ and a continuous affine mapping $\left(A_{1}\right.$ or $\left.A_{2}\right)$ is d.c. (cf. [11, Lemma 1.5]).

By Proposition 21 in [4], there exists an order-d.c. mapping $\mathbb{R} \rightarrow \ell_{2}$ which is nowhere d.c. Since the proof therein uses only the fact that the sequence $\{1 / n\}$ lies in $\ell_{2}$ but not in $\ell_{1}$, the mapping constructed in [4] (Proposition 20 and Proposition 21 for $X=\mathbb{R}$ ) is an order-d.c. mapping $F_{1}: \mathbb{R} \rightarrow \ell_{p}(1<p<\infty)$ which is nowhere d.c.

It is easy to see that, since $L_{p}(\mu)$ is infinite dimensional, there exists a countable family $\left\{E_{n}\right\}$ of pairwise disjoint $\mu$-measurable sets of a positive finite measure. Then the mapping

$$
T: \ell_{p} \rightarrow L_{p}(\mu), \quad y \mapsto T y:=\sum_{n=1}^{\infty} \frac{y(n)}{\mu\left(E_{n}\right)^{1 / p}} \chi_{E_{n}}
$$

is an order-preserving (linear) isometry. Consequently, the mapping $F_{2}:=T \circ F_{1}: \mathbb{R} \rightarrow$ $L_{p}(\mu)$ is an order-d.c. mapping which is clearly nowhere d.c.

Fix an arbitrary $0 \neq a^{*} \in X^{*}$ and define $F:=F_{2} \circ a^{*}$. Suppose that $F$ is d.c. on some open convex $C \subset X$. Fix $x_{0} \in C$ and $v \in X$ with $a^{*}(v)>0$. Then the mapping $f(t):=F\left(x_{0}+t v\right)(t \in \mathbb{R})$ is d.c. on some neighbourhood of 0 . On the other hand, $f(t)=F_{2}(\alpha+\beta t)$ where $\alpha=a^{*}\left(x_{0}\right)$ and $\beta=a^{*}(v)>0$; it clearly implies that $f$ is nowhere d.c., a contradiction.

Remark 4.4. The statement of Proposition 4.3 also holds for $p=\infty$, but the proof is slightly different. Put

$$
g_{n, r}(t):=(|n|+1)^{-1}|t-n-r| \text { for } t \in \mathbb{R}, n \in \mathbb{Z}, r \in \mathbb{Q}_{0}:=\mathbb{Q} \cap(0,1)
$$

(where $\mathbb{Z}(\mathbb{Q})$ is the set of all integers (rational numbers)). Then $g:=\left(g_{n, r}\right)_{(n, r) \in \mathbb{Z} \times \mathbb{Q}_{0}}$ is a continuous convex operator from $\mathbb{R}$ into $\ell^{\infty}\left(\mathbb{Z} \times \mathbb{Q}_{0}\right) \cong \ell^{\infty}$ which is nowhere d.c. The rest of the proof is similar to that for the case $1<p<\infty$ using an order-preserving linear isometry $T: \ell^{\infty} \rightarrow L^{\infty}(\mu)$.

\section{Jordan decomposition of finitely additive measures}

Let $\mathcal{A}$ be an algebra of subsets of a set $E$, let $Y$ be a Banach lattice and let $\mu: \mathcal{A} \rightarrow Y$ be a finitely additive measure of finite variation. The Jordan decomposition theorem for 
such $\mu$ holds in the case when $Y$ is a Nakano space (see [10] and $[\mathbf{7}]$ ). We observe that the natural decomposition procedure (essentially the same as is sketched in [7]) and a well-known result of [8] on Levi spaces imply that the Jordan decomposition theorem also holds in the more general case when $Y$ is a Levi space.

Theorem 5.1. Let $\mathcal{A}$ be an algebra of subsets of a set $E$, let $Y$ be a Banach lattice with the Levi property, and let $\mu: \mathcal{A} \rightarrow Y$ be a finitely additive measure of finite variation. Then there exist non-negative finitely additive measures $\mu_{2}$ of finite variation such that $\mu=\mu_{1}-\mu_{2}$. Moreover, if $\mu=\mu_{1}^{*}-\mu_{2}^{*}$, where $\mu_{1}^{*}, \mu_{2}^{*}$ are non-negative finitely additive measures, then $\mu_{1} \leqslant \mu_{1}^{*}$ and $\mu_{2} \leqslant \mu_{2}^{*}$.

Proof. Since $Y$ is a Levi space, by [8, Proposition 2.4.19(i)], we can choose $r>0$ such that, for each norm-bounded upper-directed set $M$ in $Y_{+}$, one has $\|\sup M\| \leqslant r \cdot \sup \{\|x\|$ : $x \in M\}$.

For each $A \in \mathcal{A}$, we put

$$
\mu_{1}(A):=\sup _{P}\{S(P)\} \in Y,
$$

where the least upper bound (in $Y$ ) is taken over all partitions $P=\left\{A_{1}, \ldots, A_{k}\right\}$ of $A$ (i.e. $A_{i} \in \mathcal{A}$ are pairwise disjoint and $A=\bigcup_{i=1}^{k} A_{i}$ ), and

$$
S(P)=\sum_{i=1}^{k}\left[\mu\left(A_{i}\right)\right]^{+} .
$$

The set of all sums $S(P)$ is clearly an upper-directed set. Since it is also norm bounded (because $\|S(P)\| \leqslant \operatorname{var}(\mu, A)$, where $\operatorname{var}(\mu, A)$ is the norm variation of $\mu$ on $A$ ), we deduce that $\mu_{1}(A)$ is well defined and

$$
\left\|\mu_{1}(A)\right\| \leqslant r \cdot \operatorname{var}(\mu, A) \quad \text { for each } A \in \mathcal{A} .
$$

It is easy to show that $\mu_{1}$ is finitely additive and $\mu \leqslant \mu_{1}$. Also, $\mu_{1}$ has finite variation by (5.1). Putting $\mu_{2}:=\mu_{1}-\mu$, we obtain the desired decomposition. The proof of the minimality is standard (cf. the proof of Theorem 3.7).

Remark 5.2. Under the assumptions of Theorem 5.1, the space $\mathcal{M}$ of all finitely additive $Y$-valued measures of finite variation on $\mathcal{A}$ is a lattice. Indeed, by Theorem 5.1, $\mu_{1}=\sup \{\mu, 0\}$ and clearly $\sup \{\mu, \nu\}=\mu+\sup \{\nu-\mu, 0\}$. On the other hand, we do not obtain that $\mathcal{M}$ (equipped with the norm $\|\mu\|=\operatorname{var}(\mu, E)$ ) is a normed lattice. If $Y$ is a Nakano space, then $\mathcal{M}$ is a Banach lattice (see [10] and [7]).

Denote by $\mathcal{A}^{*}$ the algebra of subsets of $E:=[a, b)$ generated by all intervals $[c, d) \subset E$. Then, for $\mathcal{A}=\mathcal{A}^{*}$, the assertion of Theorem 5.1 holds under the weaker assumption that $Y$ is $\sigma$-Levi. (Indeed, it is sufficient to use Remark 3.8 (iv) and the standard correspondence between finitely additive $Y$-valued measures on $\mathcal{A}^{*}$ and $Y$-valued functions on $[a, b]$.)

We do not know whether the assumption that $Y$ is $\sigma$-Levi is sufficient for some general class of algebras. 
Acknowledgements. The research of L.V. was partially supported by the Ministero dell'Università e della Ricerca Scientifica e Tecnologica of Italy. The research of L.Z. was partially supported by grant GAČR 201/03/0931 from the Grant Agency of Czech Republic and partially supported by grant MSM 113200007 from the Czech Ministry of Education.

\section{References}

1. C. D. Aliprantis AND K. C. Border, Infinite dimensional analysis-a hitchhiker's guide, 2nd edn (Springer, 1999).

2. C. D. Aliprantis and O. Burkinshaw, Locally solid Riesz spaces, Pure and Applied Mathematics, Volume 76 (Academic, 1978).

3. J. M. Borwein, Continuity and differentiability properties of convex operators, Proc. Lond. Math. Soc. 44 (1982), 420-444.

4. J. Duda, L. Veselý and L. ZajíčEK, On d.c. functions and mappings, Atti Sem. Mat. Fis. Univ. Modena 51 (2003), 111-138.

5. H. Federen, Geometric measure theory, Die Grundlehren der Mathematischen Wissenschaften, Volume 153 (Springer, 1969).

6. G. Jameson, Ordered linear spaces, Lecture Notes in Mathematics, Volume 141 (Springer, 1970).

7. B. LAVRIČ, On the modulus of cone absolutely summing operators and vector measures of bounded variation, Proc. Am. Math. Soc. 108 (1990), 479-481.

8. P. Meyer-Nieberg, Banach lattices (Springer, 1991).

9. H. H. Schaeffer, Banach lattices and positive operators, Die Grundlehren der Mathematischen Wissenschaften in Einzeldarstellung, Volume 215 (Springer, 1974).

10. K. D. Schмidt, Decomposition of vector measures in Riesz spaces and Banach lattices, Proc. Edinb. Math. Soc. 29 (1986), 23-39.

11. L. Veselý And L. ZaJíČEK, Delta-convex mappings between Banach spaces and applications, Diss. Math. (Rozprawy Mat.) 289 (1989), 1-48.

12. L. VeSELÝ AND L. ZAJÍČEK, On D.C. mappings and differences of convex operators, Acta Univ. Carolin. Math. Phys. 42 (2001), 89-97. 